\title{
IL-4 Level in Rifampicin-Sensitive and Rifampicin-Resistant Lung Tuberculosis Patients
}

\author{
Joko Susanto ${ }^{1}$, Jusak Nugraha ${ }^{1}$, Soedarsono ${ }^{2}$ \\ ${ }^{1}$ Department of Clinical Pathology, Faculty of Medicine, Airlangga University/Dr. Soetomo Hospital, Surabaya, Indonesia. E-mail: \\ pakkell@yahoo.com \\ ${ }^{2}$ Department of Pulmonology and Respirology, Faculty of Medicine, Airlangga University/Dr. Soetomo Hospital, Surabaya, Indonesia
}

\begin{abstract}
Tuberculosis remains a global health burden. Mycobacterium tuberculosis infection causes humoral and cellular responses. Macrophages of patients with pulmonary tuberculosis evolve M1 polarization that blocks infection or immunosuppressive M2, promoting tissue repair mediated by IL-4, IL-10, and IL-13. Previous research showed a decrease of IL-4R and IL-10 expression in lung macrophages of anti-TB drug resistance. A molecular test can detect rifampicin- resistance. There has been no study, which showed the difference in serum IL-4 levels in rifampicin-sensitive and rifampicin-resistant tuberculosis patients. This study aimed to determine the difference between circulating IL-4 levels in rifampicin-sensitive and rifampicin-resistant pulmonary tuberculosis patients. This cross-sectional observational study consecutively recruited subjects based on positive molecular and acid-fast bacilli microscopic examination from MDR-TB Clinic of the Dr. Soetomo Hospital between December 2018 to March 2019. Subjects were classified into a rifampicin-sensitive and rifampicin-resistant group. On ELISA measurement, IL-4 data were analyzed with SPSS version 17. Mann-Whitney $U$ test and ROC analysis tests were performed, and $p<0.05$ was significant for $\alpha=0.05$ (95\% CI). There was significant difference between rifampicin-sensitive group $(420 \pm 281 \mathrm{pg} / \mathrm{mL})$ and rifampicin-resistant group $(253 \pm 279 \mathrm{pg} / \mathrm{mL})(p=0.014)$. Receiver operating characteristics analysis showed AUC 0.70 , the sensitivity of $81.5 \%$, the specificity of $63.6 \%$, and the cut-off value of $235.6 \mathrm{pg} / \mathrm{mL}$. There was a significantly higher level of circulating IL-4 in the rifampicin-sensitive group than the rifampicin-resistant group. IL-4 level in healthy subjects should be measured as the normal value in the population. Immunology and metabolic parameters should be performed to increase sample homogeneity. Further study was also needed to understand the IL-4 role in rifampicin resistance of lung tuberculosis patients in the Indonesia population.
\end{abstract}

Keywords: IL-4, lung tuberculosis, sensitive, resistant, rifampicin

\section{INTRODUCTION}

Pulmonary tuberculosis (pulmonary TB) is a global health problem caused by the Mycobacterium tuberculosis bacilli. It is estimated that $1 / 3$ of the worldwide population worldwide is infected with M.tuberculosis, but only $5-15 \%$ of them develop active TB during their lifetime. In 2017, approximately 10 million people were infected with $T B$, and 1.6 million people died due to this disease, including Human Immunodeficiency Virus (HIV) co-infection. There are about 1 million new TB cases per year in Indonesia. According to data from the Ministry of Health in 2015, there were 117 case notification rates per 100,000 population, making Indonesia a country with the second-largest number of TB cases in the global community after India (23\%) but precedes China (10\%). ${ }^{1,2}$

The pathogenesis of tuberculosis has long been known, involving an immunological process between immune cells and humoral immunity after Mycobacterium tuberculosis attachment to the lung tissue. Lung macrophages as effector cells and antigen-presenting cells have an essential role. This cell response varies depending on the type of antigen that stimulates it. This mechanism is called polarization. Macrophage polarization in the course of TB disease includes M1 and M2. Macrophages with the $\mathrm{M} 1$ polarization type stimulate granulomatous tissue formation and a bactericidal ability that limits the spread of M.tuberculosis and are mediated by IL-1, IL-6, and IL-12. Macrophages with M2 polarization mediated by IL-4, IL-10, and IL-13 inhibit the bactericidal process, inhibit granulomatous tissue formation (immunosuppressive), and play a post-inflammatory role in tissue repair. Research by Wang et al. proved that lung macrophages in patients with anti-tuberculosis drug resistance showed a decrease in IL-10 and IL-4R expression, which was in line with the study by Butov et al. 
However, a study by Fatima et al. in India population showed that there was a significant increase of blood IL-4 levels in MDR-TB group compared to the healthy control group. ${ }^{3-6}$

The empirical rule shows that resistance to rifampin indicates resistance to all first-line anti-tuberculosis drugs. Rifampicin resistance can be identified from the polymerase chain reaction test, representing a mutation of the rpoB Mycobacterium tuberculosis gene from sputum in patients with positive AFB (acid-resistant bacilli). M2 polarization is characterized by increased IL-4R macrophage expression. ${ }^{7-10}$ This mechanism is a risk factor for anti-tuberculosis drug resistance. However, no studies prove that blood IL-4 levels are higher in patients with anti-tuberculosis drug resistance than in patients without anti-tuberculosis drug resistance. The aim of this study was to determine the differences in blood IL-4 levels of new positive AFB pulmonary tuberculosis patients with rifampin resistance and rifampin sensitivity.

\section{METHODS}

This study was an observational research with a cross-sectional design. Subjects were recruited at MDR-TB Outpatients Clinic of Dr. Soetomo Hospital, Surabaya, between December 2018 and March 2019 by consecutive sampling. A blood sample was collected at the Sampling Room of the Diagnostic Center Building of Dr. Soetomo Hospital, Surabaya. The blood sample was analyzed in the Laboratory of
Immunology and Developmental Research, Department of Clinical Pathology, Dr. Soetomo Hospital, Surabaya. All subjects were explained and already signed informed consent. The Ethics Committee has approved this study of Dr. Soetomo Hospital Surabaya with number 0802/KEPK/XI/2018. There was no conflict of interest in this study. The authors personally covered all costs of research. The research flowchart can be seen in Figure 1.

Inclusion criteria were new pulmonary patients proved by positive AFB with an age of 18 years, and subjects were recruited based on World Health Organization (WHO) criteria supported by positively detected molecular tests from sputum (Genexpert ${ }^{\circledR}$ from Cepheid $\mathrm{Co}$ ). Subjects were then classified into rifampicin-sensitive and rifampicin-resistant. In addition, subjects who already underwent a tuberculosis treatment program, subjects diagnosed with diabetes mellitus, HIV/AIDS, autoimmune, and voluntarily withdrew subjects were excluded from this study.

Venous blood was collected and centrifuged to obtain serum. The serum was stored at $-80^{\circ} \mathrm{C}$ at the latest 4 hours after blood sampling. Reagen kit consisting of human anti-IL-4 antibody-coated microplate, human standard IL-4, biotinylated detection antibody, and Avidin-horseradish peroxidase of Human IL-4 ELISA Elabscience ${ }^{\circledR}$ from Elabscience Laboratory, Houston, Texas, AS was used in this study. Optical density was read using Humareader Single Plus at a wavelength of $450 \mathrm{~nm}$.

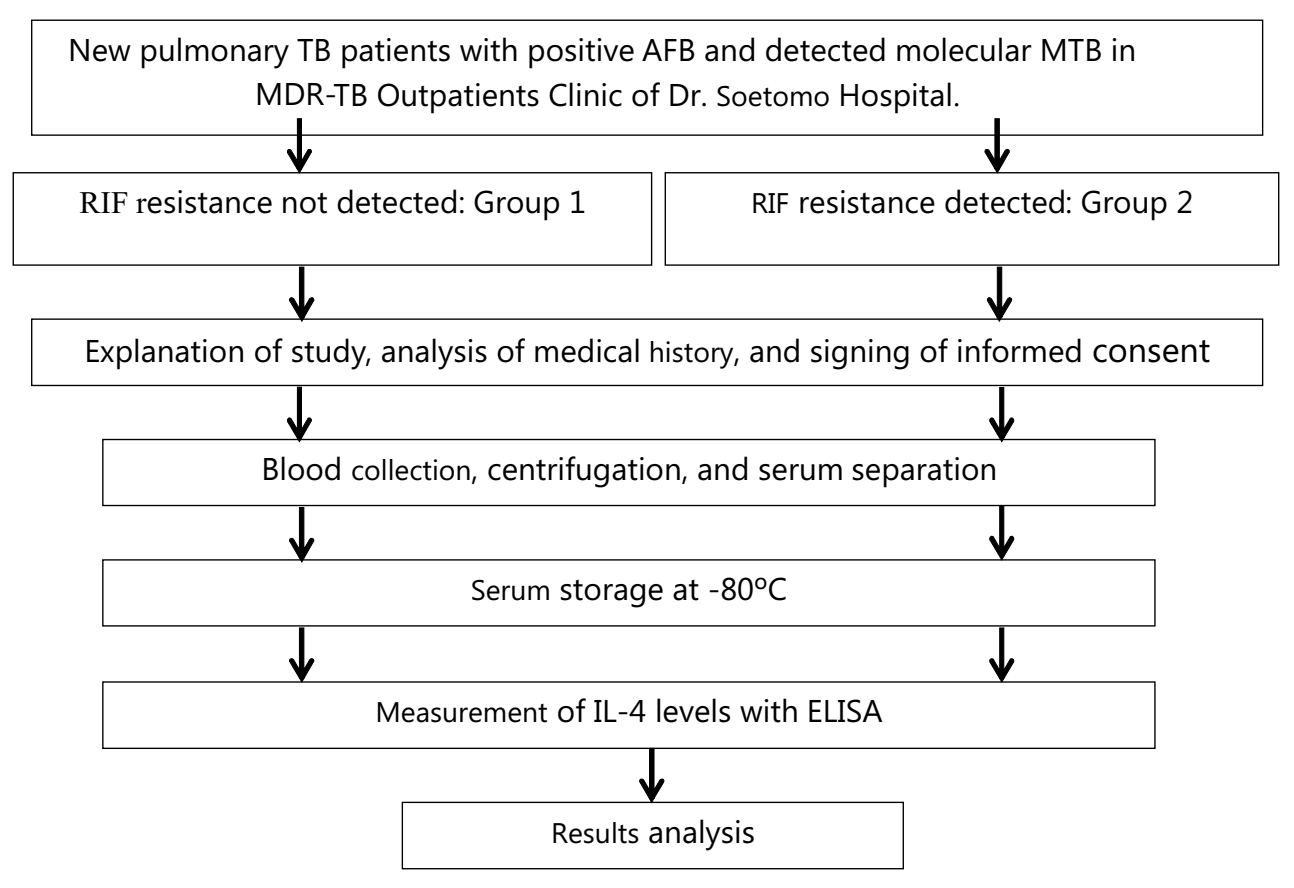

Figure 1. Research flowchart 
The results of IL-4 levels measurement were analyzed using SPSS for Windows version 17.0 from IBM. Descriptive statistics were presented to determine the subject's characteristics, including demographic, clinical data, and the results of IL-4 levels measurement. Kolmogorov-Smirnov test was used for the normality test, while the Levene homogeneity test was used for the homogeneity test. Mann-Whitney $U$ test was used because data were not normally distributed and not homogeneous. Receiver operating characteristics curves were plotted to determine diagnostic performance, sensitivity, specificity, and cut-off values for IL-4 levels. The $p$-value $<0.05$ was significant at $95 \% \mathrm{CI}$.

\section{RESULTS AND DISCUSSIONS}

A total of 49 patients were involved in this study. Rifampicin-sensitive and the rifampicin-resistant group consisted of 27 and 22 patients, respectively (Table 1).

Analysis of the Kolmogorov-Smirnov test showed abnormal distribution. Besides, the Levene test showed homogenous variance in both groups $(p>0.05)$.
Figures $2 \mathrm{a}$ and $2 \mathrm{~b}$ show distribution of IL-4 levels in each group, supported by a normal background curve. Data in both groups were not normally distributed. Figure 3 shows a box plot diagram of the distribution of IL-4 levels in both groups. The median and quartile 1 and quartile 3 in the distribution of IL-4 levels in the resistant group were lower than the rifampicin-sensitive group. There were two samples with very high values in the rifampicin-sensitive group and one sample with very high values in the rifampicin-resistant group.

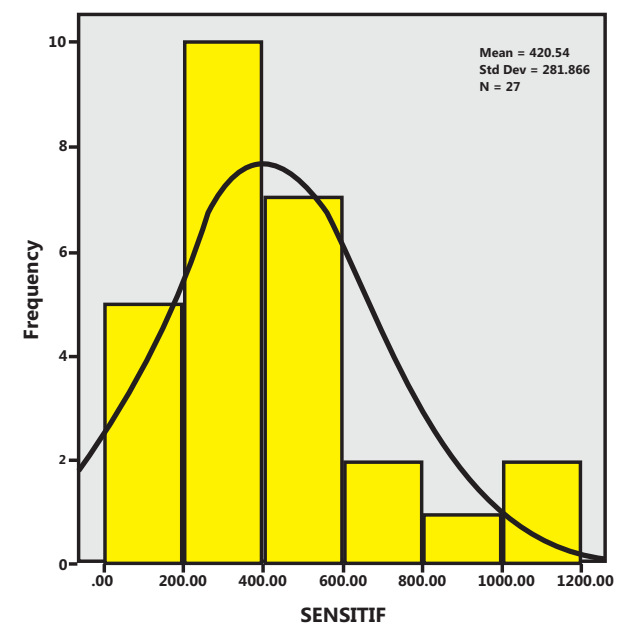

Figure 2a. Histogram of IL-4 levels in the rifampicin-sensitive group

Table 1. Characteristics of subjects and results of difference test

\begin{tabular}{|c|c|c|c|}
\hline Characteristics & Sensitive $(n=27)$ & Resistant (n=22) & p-value \\
\hline Age $($ mean $\pm S D)$ & $33(20)$ & $49.5(13.5)$ & $0.287^{*}$ \\
\hline Gender (\%) & & & $0.181^{*}$ \\
\hline Male & $12(24 \%)$ & $14(29 \%)$ & \\
\hline Female & 15(31\%) & $8(16 \%)$ & \\
\hline \multicolumn{4}{|l|}{ Clinical signs (\%) } \\
\hline Cough $>2$ weeks & $26(53 \%)$ & $22(45 \%)$ & $0.362^{*}$ \\
\hline Hemoptysis & $2(4 \%)$ & $6(12 \%)$ & $0.061^{*}$ \\
\hline Fever & $7(14 \%)$ & $8(16 \%)$ & $0.915^{*}$ \\
\hline Night sweats & $10(37 \%)$ & $13(60 \%)$ & $0.124^{*}$ \\
\hline Decreased body weight & $15(55 \%)$ & $11(50 \%)$ & $0.482^{*}$ \\
\hline Dyspnea & $19(70 \%)$ & $12(55 \%)$ & $0.253^{*}$ \\
\hline Chest pain & $6(22 \%)$ & $4(18 \%)$ & $0.518^{*}$ \\
\hline Smoking & $8(30 \%)$ & $8(36 \%)$ & $0.617^{*}$ \\
\hline Alcohol & $2(7 \%)$ & $1(4 \%)$ & $0.678^{*}$ \\
\hline Enlarged gland & $1(4 \%)$ & - & $0.362^{*}$ \\
\hline \multicolumn{4}{|l|}{ BTA (\%) } \\
\hline+1 & $23(85 \%)$ & $11(50 \%)$ & $0.008^{*}$ \\
\hline+2 & $4(15 \%)$ & $10(45 \%)$ & $0.018^{*}$ \\
\hline+3 & - & $1(5 \%)$ & - \\
\hline \multicolumn{4}{|l|}{ IL-4 (ng/mL) } \\
\hline Mean $\pm S D$ & $420,54 \pm 281,56$ & $253,64 \pm 279,30$ & \\
\hline Median (IQR) & $348,70(287,95)$ & $133,72(366,66)$ & $0.014^{\star *}$ \\
\hline
\end{tabular}

${ }^{\star}$ Cross-tabulation analysis ${ }^{*}$ Mann-Whitney test 


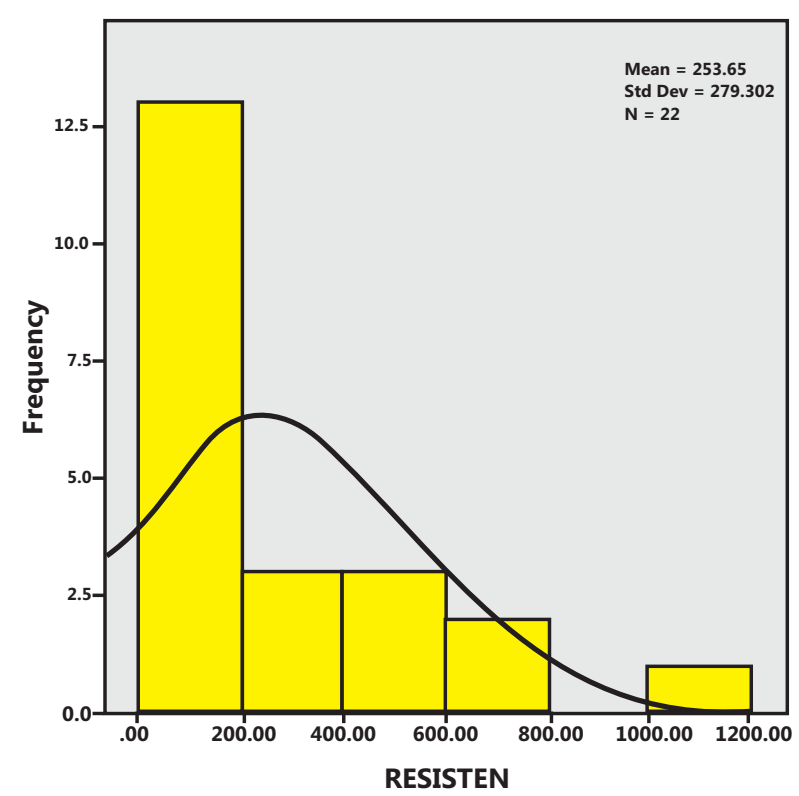

Figure 2b. Histogram of IL-4 levels in the rifampicin-resistant group

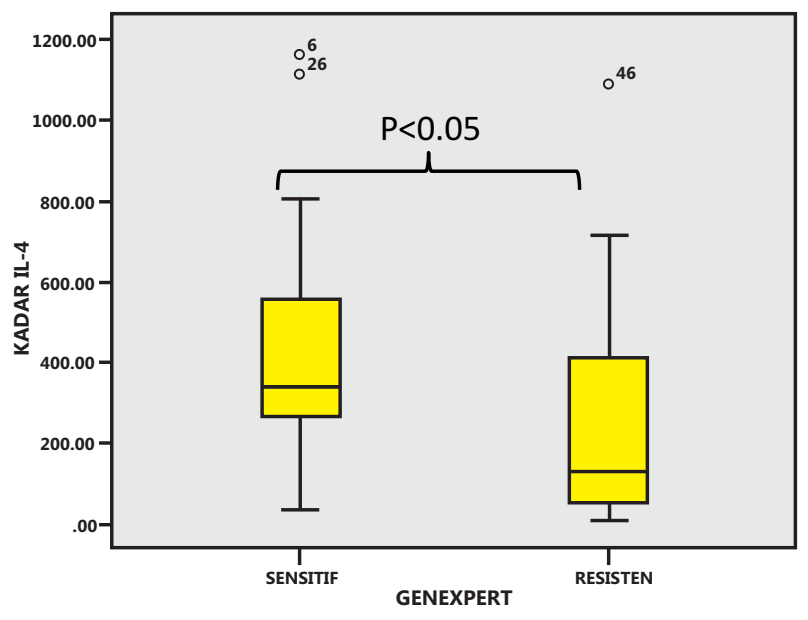

Figure 3. Distribution of IL-4 levels in both groups in the boxplot diagram

Because the data from the two groups were not normally distributed in spite of homogeneous variance, parametric statistical tests could not be performed. Receiver operating characteristics curve analysis was used to determine diagnostic performance. Figure 4 shows the ROC curve with an area under the curve of 0.70 , which was categorized as fair. At a cut-off value of $235.3 \mathrm{pg} / \mathrm{mL}$, the diagnostic sensitivity and diagnostic specificity was $81.5 \%$ and $63.6 \%$ with $p$-value $<0.05$ (95\% CI).

Due to abnormal distribution and heterogeneity of data, a parametric statistical test was not used.

There were no significant differences in age, gender, and clinical data among 49 subjects enrolled in this study. This showed that these three things could not be used as a standard as a risk factor for

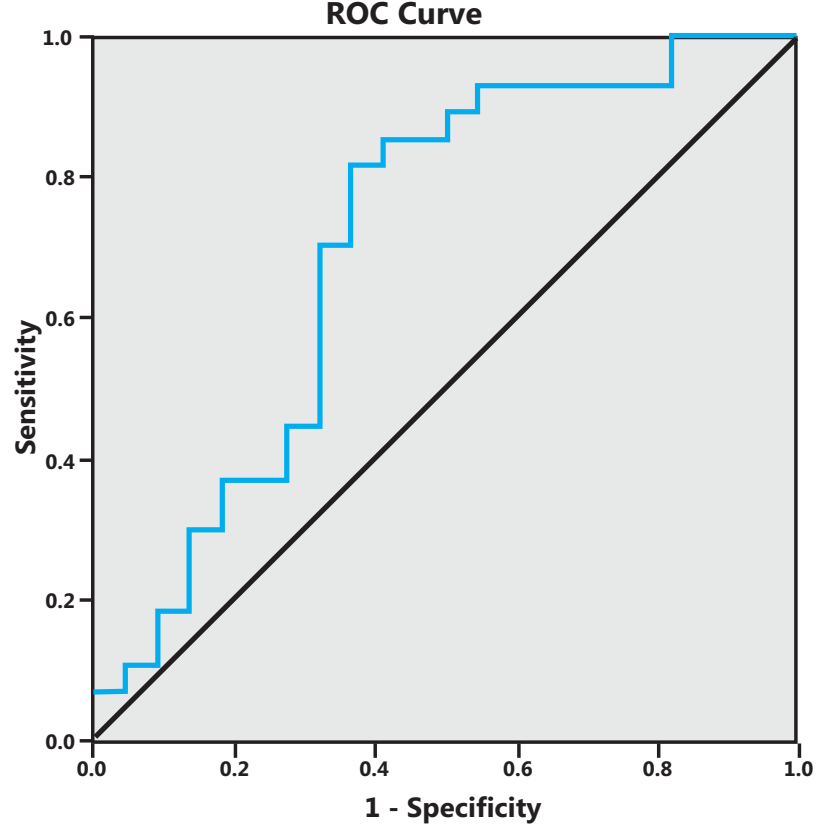

Figure 4. ROC curve of difference in IL-4 levels between both groups

rifampin resistance and was not related to serum IL-4 levels.

In contrast, the number of acid-fast bacilli in the two groups were significantly different. This fact suggested that rifampicin resistance and blood IL-4 levels were related to the degree of bacteriology, as indicated by the number of bacteria in sputum.

IL-4 levels in the two groups were not normally distributed based on the normality test. Multi-factors can cause various distribution of IL-4 levels. These results required further study because there was no evaluation of those multi-factors in this study. No additional laboratory tests, such as metabolic parameters and immunology tests, were among the limitations of this study. This finding was because metabolic disorders or immunological disorders can affect IL-4 production.

Despite the abnormal distribution of IL-4 levels, different tests using the Mann-Whitney $U$ test showed significant differences. These findings collectively indicated similar mechanisms of body response to Mycobacterium tuberculosis infection in each group. However, this fact required further study regarding the type of polarization of lung macrophages in the subject of this study.

Although the results of this study were different from the study by Fatima et al., the results of this study were in line with the study by Butov et al. in the Ukrainian population, which showed that serum IL-4 levels of Multidrug-Resistant (MDR) pulmonary TB 
patients were significantly lower than non-MDR-TB both before treatment and after two months of treatment. ${ }^{6,10}$ The IL-4 levels in both groups were also considerably lower than the healthy group. The study also showed that C589T polymorphism of the IL-4 gene is significant in the inflammatory mechanism in TB patients. These findings also explain the higher rates of Th2 lymphocyte activation and Th1 lymphocyte stabilization in patients with non-MDR-TB compared to MDR-TB. It suggests the role of immunogenetics in the pathophysiology of tuberculosis. ${ }^{10}$

The diagnostic performance of the results of this study was relatively good. An AUC value of 0.70 indicated an adequate diagnostic performance. It showed that differences in IL-4 levels in both groups were not caused by chance or randomness, but that difference was related to differences in the body's response to Mycobacterium tuberculosis. At the optimal cut-off value of $253.3 \mathrm{pg} / \mathrm{mL}$, the highest sensitivity and specificity were $81.5 \%$ and $63.6 \%$, respectively ( $p<0.05,95 \% \mathrm{CI})$. Therefore, IL-4 levels were adequately sensitive but not adequately specific to differentiate the anti-tuberculosis drug's resistance status.

No healthy subjects as normal controls in this study were another limitation of this study, in contrast, to study by Butov et al. and Fatima et al. Therefore, this study's results were unable to describe blood IL-4 levels in healthy people. Further research was needed to explain the pathophysiology of tuberculosis in the Indonesian population. ${ }^{6.10}$

\section{CONCLUSIONS AND SUGESSTIONS}

IL-4 levels in the rifampin-sensitive pulmonary TB group were significantly different from the rifampin-resistant TB group. The IL-4 level in the rifampicin-sensitive group was significantly higher than the rifampicin-resistant group at a cut-off value of $235.3 \mathrm{pg} / \mathrm{mL}$. Significant differences in AFB 1+ and $2+$ were found between both groups. However, there were no significant differences in clinical and demographic data.

Measurement of IL-4 levels in healthy subjects (healthy controls) was also needed to determine the population's normal value. Immunological and metabolic parameters were required to result in a more homogeneous sample. It was also necessary to carry out further research on the role of IL-4 in patients with rifampicin-sensitive and rifampicin-resistant pulmonary TB in the Indonesian population.

\section{REFERENCES}

1. World Health Organization. Publication of tuberculosis. [Internet]. 2019. Cited from https://www.who.int/news-room/fact-sheets/ detail/tuberculosis. [accessed 30 June, 2019].

2. Pusat Data dan Informasi (Pusdatin) Kementerian Kesehatan Republik Indonesia. Tuberkulosis: Temukan, obati sampai sembuh. Booklet Infodatin. [Internet]. 2018. Cited from http://www.pusdatin. kemkes.go.id/article/view/16080300002 /-tuberkulosis-2016.html. [accessed 9 September, 2018].

3. Huang Z, Luo Q, Guo Y, Chen J, Xiong, et al. Mycobacterium tuberculosis-induced polarization of human macrophage orchestrates the formation and development of tuberculous granulomas in-vitro. PLos One, 2015; 10(6): e0129744.

4. Lugo-Villarino G, Verollet C, Maridonneau-Parini I, Neyrolles O. Macrophage polarization: Convergence point targetted by Mycobacterium tuberculosis and HIV. Frontiers in Immunology, 2011; 2: 43. Cited from www.ncbi.nlm.nih.gov/pmc/articles/PMC3342390/ [accessed 9 September, 2018].

5. Wang S, Zhang J, Sui L, Xu H, Piao Q, et al. Antibiotics induce polarization of pleural macrophages to M2-like phenotype in patients with tuberculous pleuritis. Nature Scientific Reports, 2017; 7: 14982. Cited from https://www.nature.com/articles/s41598-017-14808-9. [accessed 12 August, 2018].

6. Fatima N, Shameem M, Nabeela, Khan PA, Khan HM. Correlation of serum IL-4 levels in new and MDR tuberculosis patients. American Journal of Clinical and Experimental Medicine, 2015; 3(1): 48-51.

7. Labonte AC, Tosello TAC, Hahn YS. The role of macrophage polarization in infectious and inflammatory diseases. Molecules and Cells Journal, 2014; 37(4): 275-285.

8. Vidyarani $M$, Selvaraj $P$, Anand SP, Jawahar MS, Adhilaksmi AR, Narayanan PR. Interferon-gamma (IFNY) and interleukin-4 (IL-4) gene variants and cytokine levels in pulmonary tuberculosis. The Indian Journal of Medical Research, 2006; 124(4): 403-10.

9. Ordway DJ, Costa L, Martins M, Silveira H, Amaral L, et al. Increased interleukin-4 production by CD8 and $\mathrm{y} \delta 1 \mathrm{~T}$-cells in healthcare workers is associated with the subsequent development of active tuberculosis. The Journal of Infectious Disease, 2004; 190(4): 756-766.

10. Butov DO, Kuzhko MM, Makeeva NI, Butova TS, Stepanenko HL, Dudnyk AB. Association of interleukins genes polymorphisms with multidrug-resistant tuberculosis in Ukrainian population. Advances in Respiratory Medicine, 2016; 84(3): 168-173. 